\title{
Neuropsychiatric Adverse Reactions to Mefloquine: a Systematic Comparison of Prescribing and Patient Safety Guidance in the US, UK, Ireland, Australia, New Zealand, and Canada
}

Remington L. Nevin • Aricia M. Byrd

Received: April 19, 2016/Published online: May 30, 2016

(c) The Author(s) 2016. This article is published with open access at Springerlink.com

\begin{abstract}
Introduction: The antimalarial drug mefloquine (MQ) is associated with neuropsychiatric adverse reactions, some of which may predict the development of more serious effects. Although prescribing guidance in the United States drug label (DL) recommends to discontinue $\mathrm{MQ}$ at the onset of neuropsychiatric symptoms, only certain reactions are listed in both the DL and the corresponding patient medication guide with a recommendation to discontinue or to consult a physician should they occur. To identify possible prodromal reactions for which there is
\end{abstract}

Enhanced content To view enhanced content for this article go to http://www.medengine.com/Redeem/25D4 F0600B7DFC9C.

R. L. Nevin $(\bowtie)$

Department of Mental Health, Johns Hopkins

Bloomberg School of Public Health, Baltimore, MD,

USA

e-mail: rnevin@jhu.edu

A. M. Byrd

Trinity School of Medicine, Kingstown, Saint

Vincent and the Grenadines complete or partial agreement in prescribing and patient recommendations, a systematic comparison of international drug safety labeling was performed.

Methods: The full text of each DL and medication guide (or equivalent) from six primarily English-speaking countries was reviewed to identify specific reactions with corresponding recommendations in drug safety labeling. Percentage agreement across the countries in corresponding recommendations was determined by MedDRA ${ }^{\circledR}$ high level group term (HLGT).

Results: Recommendations were found for reactions in 22 neuropsychiatric HLGTs. Complete or partial international agreement was found for reactions in 11 (50\%) HLGTs.

Conclusion: This analysis suggests opportunities for physicians to improve patient counseling and for international drug regulators to clarify language in MQ safety labeling to reflect national risk-benefit considerations.

Keywords: Medication guide; Mefloquine; Neurologic; Psychiatric; Side effects 


\section{INTRODUCTION}

Mefloquine (MQ) is a synthetic quinoline-derivative antimalarial drug structurally related to quinine that exhibits idiosyncratic central nervous system toxicity [1]. In double blinded studies, a range of neuropsychiatric adverse reactions-including strange or vivid dreams, dizziness, vertigo, concentration impairment, anxiety, and depression-are reported by $29-77 \%$ of MQ users at prophylactic doses of $250 \mathrm{mg}$ weekly $[2,3]$. Neuropsychiatric adverse reactions may occur early during use-frequently within the first three doses-and may even occur after only a single dose [4, 5].

As evidenced by a recent retrospective cohort study, among those reporting adverse reactions to $\mathrm{MQ}, 21 \%$ of those reporting nightmares and $33 \%$ of those reporting cognitive dysfunction identified these adverse reactions as persisting over 3 years after use [6]. A boxed warning added to the United States (US) drug label (DL) in 2013 emphasizes that MQ may cause "neuropsychiatric adverse reactions that can persist after mefloquine has been discontinued". Prescribing guidance in the US DL now recommends to discontinue (DC) MQ at the onset of neuropsychiatric symptoms, as certain of these may suggest an individual risk of "more serious psychiatric disturbances or neurologic adverse reactions" that could occur with continued use of the drug. The US DL now cautions that psychiatric reactions "ranging from anxiety, paranoia, and depression to hallucinations and psychotic behavior can occur with mefloquine use" and "have been reported to continue for months or years after mefloquine has been stopped". The US DL now also cautions that certain neurological reactions "have been reported to be permanent in some cases" [7].
The highly prescriptive safety guidance in the current DL reflects its gradual evolution over the prior quarter century. At the time of the US licensing of MQ in 1989, the original DL instructed physicians only that, "[d]uring prophylactic use, if signs of unexplained anxiety, depression, restlessness or confusion are noticed, these may be considered prodromal to a more serious event. In these cases, the drug must be discontinued" [8]. This language was subtly updated in 2002, changing the previously exclusive list of prodromal reactions to an illustrative list by stating, "if psychiatric symptoms such as [emphasis added] acute anxiety, depression, restlessness or confusion occur, these may be considered prodromal to a more serious event. In these cases, the drug must be discontinued and an alternative medication should be substituted" [9].

The 2002 DL update introduced potential ambiguity as to whether US physicians were to counsel patients that the onset of any psychiatric symptom should be considered prodromal and prompt DC, or merely that those explicitly listed and similar reactions should be considered prodromal and prompt DC. The 2013 DL update likewise noted, "[d]uring prophylactic use, the occurrence of psychiatric symptoms such as acute anxiety, depression, restlessness or confusion suggest a risk for more serious psychiatric disturbances or neurologic adverse reactions [emphasis added]. In these cases, the drug should be discontinued and an alternative medication should be substituted". The 2013 boxed warning addressed any remaining potential ambiguity in this illustrative list by emphasizing simply, "During prophylactic use, if psychiatric or neurologic symptoms occur, the drug should be discontinued and an alternative medication should be substituted" [7]. 
The reasoning that prompted these changes was not made explicitly clear in the 2013 DL or in the accompanying US Food and Drug Administration (FDA) drug safety communication [10]. However, the following year in a 2014 pharmacovigilance review, the European Medicines Agency (EMA) concluded "a causal relationship between mefloquine and the occurrence of long lasting and even persistent neuropsychiatric effects", and noted a "strong suspicion" that MQ could in some cases cause "permanent brain damage" [11]. The EMA noted that no "specific risk factors" could be identified for these effects, and concluded, "[f]or that reason, only the advice-to stop taking mefloquine if neuropsychiatric reactions or changes to their mental state occur-can be given as a precautionary measure" [11].

In the US, in the case of certain drugs "that pose a serious and significant public health concern", the FDA may require specific safety guidance be communicated directly to patients in the form of a medication guide (MG) provided at the time of dispensing, which complements counseling received by the patient at the time of prescribing [12]. The FDA requires a MG when it determines patient adherence to directions for use are considered crucial to a drug's effectiveness; when the drug has serious risks relative to benefits; or when patient safety guidance in the MG could help prevent "serious adverse effects" [12]. Consistent with this final rationale, the MG for MQ was first required by the FDA in 2003 [13].

The current US MG explicitly lists certain psychiatric or neurologic adverse reactions for which patients are recommended to consult with a physician or healthcare provider (CP) prior to taking their next dose. Although the US boxed warning clearly recommends DC "if psychiatric or neurologic symptoms occur", as is the case with the MG, only certain specific neurologic or psychiatric adverse reactions are explicitly listed in the current US DL with a recommendation to DC [7].

The rationale for the specific choice of listed adverse reactions for which the US MG and DL are in correspondence in recommending DC or $\mathrm{CP}$ is not clear. The choice may reflect consensus decision making between the FDA and the drug's manufacturers on those prodromal or precursor adverse reactions that most "suggest a risk for more serious psychiatric disturbances or neurologic adverse reactions", and which should, therefore, be specifically highlighted in light of risk-benefit considerations particular to the US regulatory and legal environment. Such decision making may include a consideration of the predictive value of a particular prodromal or precursor adverse reaction in foretelling more serious reactions with continued use of the drug.

In other countries, based on the results of independent regulatory decision making and potentially differing risk-benefit considerations, the particular choice of listed neurologic and psychiatric adverse reactions for which DC or $\mathrm{CP}$ may be recommended may differ from those of the US. International agreement in specific listed neurologic and psychiatric adverse reactions for which MQ safety guidance recommends DC or CP may, therefore, reflect agreement on the strength of the evidence and the consistency of risk-benefit decision making motivating those recommendations.

To compare and contrast current international recommendations for actions to be taken in response to specific MQ neurologic and psychiatric adverse reactions, and to identify those categories of adverse reaction for which there is international agreement in listing such recommendations, a systematic comparison was 
performed of current prescribing and patient safety guidance in the US and five other primarily English-speaking countries: the United Kingdom (UK), Ireland (IRL), Australia (AUS), New Zealand (NZ), and Canada (CAN).

\section{METHODS}

\section{Prescribing and Patient Safety Documents}

Prescribing safety guidance in the UK and IRL is provided in a document referred to as a Summary of Product Characteristics; in AUS as a product information; in NZ as a Data Sheet; and in CAN as a product monograph-all of which are herein referred to as a DL. Although not always comparably mandated in each country, patient safety guidance similar to that provided in the US MG is provided in AUS and $\mathrm{NZ}$ in a document referred to as the Consumer Medicine Information; in IRL as the Patient Leaflet; in the UK as the package leaflet; and in CAN as information for the patient-all of which are herein referred to as a MG.

The most recent MQ DL and MG as of December 2015 were identified through a search of the websites of national drug regulators and drug manufacturers in each of the six countries. The DL and MG were retrieved for the innovator product (marketed as Lariam $^{\circledR}$, Roche Products Ltd.) in those countries where the innovator product remained marketed; and for the generic product in those countries where the innovator product had been withdrawn.

\section{Review of Prescribing and Patient Guidance}

The full text of each DL and MG was then reviewed by two clinicians to identify recommendations for actions to be taken in response to specific listed neurologic or psychiatric adverse reactions. Disagreements between the clinicians during review were resolved by consensus.

Where a recommendation stated the drug "should" or "must" be discontinued or stopped at the onset of a listed adverse reaction, this was categorized as a recommendation to DC. Where the text did not include an explicit direction to DC or where the text suggested only that it "may be necessary to stop", but included a recommendation to "consult immediately" or "consult" a healthcare provider at the onset of a listed adverse reaction, this was categorized as a recommendation to $\mathrm{CP}$. Where contradictory guidance for a listed adverse reaction appeared in different locations in the text, a recommendation to DC took precedence.

Where one or more adverse reactions were listed ambiguously within a paragraph that contained a particular recommendation associated with a smaller list of adverse reactions or a more general description of an adverse reaction, the recommendation was deemed to apply to that term. For example, based on the following paragraph in the US DL, a recommendation to DC was deemed to apply to the adverse reactions dizziness, vertigo, tinnitus, and loss of balance.

"Neurologic symptoms such as dizziness or vertigo, tinnitus, and loss of balance have been reported. These adverse reactions may occur early in the course of mefloquine use and in some cases have been reported to continue for months or years after mefloquine has been stopped. Dizziness or vertigo, tinnitus, and loss of balance have been reported to be permanent in some cases. During prophylactic use, if neurologic symptoms occur, the drug 
should be discontinued and an alternative medication should be substituted" [7].

Where the text referred explicitly to an adverse reaction occurring only in the context of a pre-existing condition or contraindication, this was not included in this analysis. Similarly, if an adverse reaction was described only in a section of the text describing guidance applicable prior to starting $\mathrm{MQ}$, without referencing explicitly that the adverse reaction could also be caused by MQ, it was not included in this analysis. Likewise, if an adverse reaction appeared only in a table, without an explicit inclusive reference in the text to a specific recommendation, it was also not included in this analysis.

Adverse reactions were considered as neurologic or psychiatric according to the Medical Dictionary for Regulatory Activities $\left(\right.$ MedDRA $^{\circledR}$, version 18.1) [14] if the MedDRA lowest level term (LLT) matching the adverse reaction was primarily or secondarily categorized within either the MedDRA nervous system or psychiatric disorders system organ class (SOC). For example, the LLT dizziness, categorized multiaxially within the MedDRA under the SOCs cardiac disorders, vascular disorders, and nervous system disorders, was considered neurologic for the purposes of this analysis. Where a listed LLT could be considered both psychiatric and neurologic, it was considered psychiatric. For example, the LLT insomnia, categorized multiaxially within the MedDRA under the SOCs psychiatric disorders and nervous system disorders, was considered psychiatric. Where a particular adverse reaction-such as when expressed in consumer language-did not explicitly match an LLT, the closest relevant lexical or conceptual variant to MedDRA terminology was substituted. For example, the MedDRA LLT "masked facies" was substituted for the adverse reaction "difficulties with facial expression". Similarly, the MedDRA LLT "restless" was substituted for the adverse reaction "feeling restless". As similar or equivalent adverse reactions may be reported by a range of terminology, all LLTs were grouped according to their MedDRA preferred term (PT). For example, "restlessness" and "feeling restless" were grouped together on the basis of their common PT "restlessness".

\section{International Agreement in Corresponding Prescribing and Patient Guidance}

Adverse reactions were organized according to their MedDRA highest level grouping term (HLGT). Those countries, whose MG and DL both included a recommendation either to DC or CP at the onset of a PT, were deemed to be in correspondence for that adverse reaction. Countries in correspondence for one or more PT within each HLGT were identified, and for each HLGT, the percentage agreement across all six countries of the corresponding DL and MG recommendations to DC or CP for one or more PT was determined. HLGTs for which corresponding MG and DL recommendations to $\mathrm{DC}$ or $\mathrm{CP}$ for one or more PT were in agreement for all six countries were deemed to be in complete international agreement. HLGTs for which corresponding $\mathrm{MG}$ and DL recommendations to $\mathrm{DC}$ or $\mathrm{CP}$ for one or more PT were in agreement for two or more countries were deemed to be in partial international agreement.

\section{Compliance with Ethics Guidelines}

This article is based on previously conducted studies and does not involve any new studies of 
human or animal subjects performed by any of the authors.

\section{RESULTS}

At the time of analysis, the drug remained licensed in all six countries, although the innovator product had been withdrawn from the US as of 2011 and from CAN as of 2013 [15, 16]. The most recent generic US DL and MG available were each dated June 2013, and the most recent generic CAN DL and MG available were each dated March 2011. Lariam-branded DL and MG were available for the UK, IRL, AUS, and NZ. The UK DL was dated December 2015, and the MG was dated April 2015. The IRL DL was dated June 2015, and the MG was dated May 2015. The AUS DL and MG were both dated November 2014, while the NZ DL and MG were both dated August 2014.

In addition to being explicitly recommended by the US DL, the DLs of the UK and IRL also explicitly recommended DC at the onset of general neurologic or psychiatric symptoms. Echoing the language in the earlier EMA document [11], the UK and IRL DL recommended:

"If neuropsychiatric reactions or changes to the mental state occur during mefloquine chemoprophylaxis [emphasis added], the patient should be advised to stop taking mefloquine and seek medical advice immediately, so that mefloquine can be replaced by alternative malaria prevention medication".

Among the six DLs and six MGs, additional patient and prescribing guidance was identified for specific adverse reactions within 22 distinct neuropsychiatric HLGTs, comprising 14 psychiatric HLGTs (Table 1) and 8 neurologic HLGTs (Table 2). There was complete agreement across all six countries in corresponding MG and DL recommendations to DC or CP for adverse reactions within four (18\%) of the HLGTs. These HLGTs were anxiety disorders and symptoms, changes in physical activity, depressed mood disorders and disturbances, and deliria (including confusion). There was partial agreement across three of the six countries (US, $\mathrm{UK}$, and IRL) in corresponding MG and DL recommendations to DC or CP for adverse reactions within three (14\%) additional HLGTs. These HLGTs were disturbances in thinking and perception, personality disorders and disturbances in behavior, and suicidal and self-injurious behaviors not elsewhere classified (NEC). There was also partial agreement across two of the six countries (UK and IRL) in corresponding MG and DL recommendations to DC or CP for adverse reactions within four (18\%) additional HLGTs. These were neuromuscular disorders, schizophrenia, and other psychotic disorders, sleep disorders and disturbances, and peripheral neuropathies.

In a single country (US), there was also corresponding MG and DL guidance to DC or $\mathrm{CP}$ for adverse reactions within two (9\%) additional HLGTs. These were cranial nerve disorders (excluding neoplasms) and neurological disorders NEC. Among adverse reactions within nine (41\%) additional HLGTs, although patient guidance in the MG from at least two countries each recommended CP, there was no corresponding guidance provided to physicians for these specific adverse reactions in the DL (Table 3).

\section{DISCUSSION}

\section{Agreement in Prescribing and Patient Guidance}

This analysis finds complete international agreement across the six countries in corresponding prescribing and patient safety 
Table 1 Psychiatric adverse reactions to mefloquine, prescribing and patient guidance, by country

\begin{tabular}{|c|c|c|c|c|c|c|}
\hline Adverse reaction HLGT (bold) and LLT & US & UK & IRE & AUS & $\mathrm{NZ}$ & CAN \\
\hline & $\overline{\mathrm{DL}} \quad \mathrm{MG}$ & $\overline{\mathrm{DL}} \quad \mathrm{MG}$ & $\overline{\mathrm{DL}} \quad \mathrm{MG}$ & $\overline{\mathrm{DL}} \quad \mathrm{MG}$ & MG & DL \\
\hline
\end{tabular}

Anxiety disorders and symptoms

Agitation

DC

DC

$\mathrm{CP}$

$\mathrm{CP}$

(Acute/severe) anxiety/anxiety disorders/feeling

$\mathrm{DC} \mathrm{CP}$

DC DC

DC DC

DC $\quad$ CP $\quad$ DC $\quad$ CP $\quad$ DC $\quad$ CP anxious

Excitement

$\begin{array}{llll} & & \mathrm{CP} & \mathrm{CP} \\ & & \mathrm{CP} & \mathrm{CP} \\ \mathrm{DC} & \mathrm{DC} & \mathrm{CP} & \mathrm{CP}\end{array}$

Nervousness/feeling nervous

Panic attack

Changes in physical activity

Restlessness/feeling restless

$\begin{array}{llllllllllll}\text { DC } & \text { CP } & \text { DC } & \text { DC } & \text { DC } & \text { DC } & \text { DC } & \text { CP } & \text { DC } & \text { CP } & \text { DC } & \text { CP }\end{array}$

Cognitive and attention disorders and disturbances

Disturbance with attention

DC DC

Deliria (including confusion)

Confusion/feeling confused

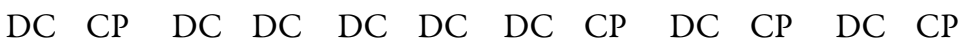

Dementia and amnestic conditions

Forgetfulness

CP $\quad$ CP

Depressed mood disorders and disturbances

Depression

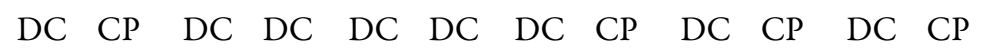

Disturbances in thinking and perception

Hallucinations

DC CP DC DC DC DC

$\begin{array}{ll}\mathrm{CP} & \mathrm{CP} \\ \mathrm{CP} & \mathrm{CP} \\ \mathrm{CP} & \end{array}$

Irrational ideas ${ }^{\mathrm{a}}$

Strange or disturbing thoughts ${ }^{\mathrm{b}}$

Mood disorder and disturbances NEC

Irritability

Unusual changes in mood/change in mood/ strange $\operatorname{mood}^{c}$

Psychiatric and behavioral symptoms NEC

Unusual behavior $^{\mathrm{d}}$

CP DC DC

Personality disorders and disturbances in behavior

Aggression

DC DC

$\mathrm{CP}$

CP

Feeling of mistrust towards others/paranoia

DC CP DC DC DC DC 
Table 1 continued

\begin{tabular}{|c|c|c|c|c|c|c|}
\hline Adverse reaction HLGT (bold) and LLT & US & $\mathbf{U K}$ & IRE & AUS & $\mathbf{N Z}$ & CAN \\
\hline & MG & MG & MG & MG & MG & DL \\
\hline
\end{tabular}

Psychiatric disorders NEC

Changes to the mental state ${ }^{\mathrm{e}}$

DC DC

Schizophrenia and other psychotic disorders

Losing touch with reality/psychosis

DC DC DC DC

Psychotic behavior

DC

\section{Sleep disorders and disturbances}

Abnormal dreams/strange dreams

Insomnia/(unable to/inability to) sleep/

difficulty sleeping

Nightmares/bad dreams

Sleeping problems ${ }^{\mathrm{f}}$

DC DC DC DC

Suicidal and self-injurious behaviors NEC

Attempted suicide

DC DC DC DC

Suicidal thoughts/suicidal ideation/think about suicide $^{\mathrm{g}}$

DC DC DC DC

CP CP CP

CP

DC
CP
CP

CP

CP

Self-endangering behavior ${ }^{\mathrm{h}}$

DC DC DC DC

AUS Australia, CAN Canada, $C P$ consult physician, $D C$ discontinue drug, $D L$ drug label (or national equivalent), $H L G T$ MedDRA high level group term, IRL Ireland, $L L T$ MedDRA lowest level term, $M G$ medication guide (or national equivalent), $N E C$ not elsewhere classified, $N Z$ New Zealand, UK United Kingdom, US United States

${ }^{a}$ LLT thinking irrational

b LLT thinking abnormal

c LLT mood change

d LLT abnormal behavior

e LLT mental state abnormal

${ }^{f}$ LLT sleep disorder

g LLT suicidal ideation

h LLT self-injurious behavior

guidance to $\mathrm{CP}$ or $\mathrm{DC} \mathrm{MQ}$ in response to specific psychiatric adverse reactions within four HLGTs. These include the common adverse reactions of depression and anxiety within the HLGTs depressed mood disorders and disturbances, and anxiety disorders and symptoms. The DLs of the UK, IRL, AUS, and NZ each describe anxiety and depression occurring in $\geq 1 / 100-1 / 10$ of prophylactic users.
In contrast, this analysis finds only partial international agreement across two or more countries in corresponding prescribing and patient safety guidance to CP or DC in response to specific neurologic and psychiatric adverse reactions within an additional seven HLGTs. These include the very common adverse reaction of abnormal dreams, included within the HLGT sleep disorders and disturbances. The 
Table 2 Neurologic adverse reactions to mefloquine, prescribing and patient guidance, by country

\begin{tabular}{|c|c|c|c|c|c|c|}
\hline Adverse reaction HLGT (bold) and LLT & US & UK & IRE & AUS & $\mathrm{NZ}$ & CAN \\
\hline & DL MG & DL MG & DL MG & DL MG & MG & DL $\quad M$ \\
\hline
\end{tabular}

Cranial nerve disorders (excluding neoplasms)

Difficulties with sense of smell or taste ${ }^{a}$

Changes to hearing/difficulties hearing/hearing disturbances $^{\mathrm{b}}$

Things seem to sound too loud ${ }^{c}$

Ringing in ears/tinnitus

DC CP

$\mathrm{CP}$

$\mathrm{CP}$

$\mathrm{CP}$

CP

Headaches

Headache

Movement disorders (including parkinsonism)

Shaking/tremors

Difficulties with facial expression ${ }^{\mathrm{d}}$

Difficulties with head turning

Clumsiness

\section{Neurological disorders of the eye}

Blurred vision

\section{Neurological disorders NEC}

Burning $^{f}$

Difficulties talking ${ }^{\mathrm{g}}$

Difficulties with balance/loss of balance ${ }^{\mathrm{h}}$

Difficulties with eye movement ${ }^{i}$

Difficulties with facial sensation ${ }^{j}$

Difficulties with tongue movement ${ }^{\mathrm{k}}$

Dizziness/light-headedness

Fainting/loss of consciousness

Numbness/numbness in the hands or feet/ tingling

Pain ${ }^{1}$

Pins and needles

Unsteadiness

Vertigo

\section{Neuromuscular disorders}

Weakness $^{\mathrm{m}}$
DC CP

DC CP

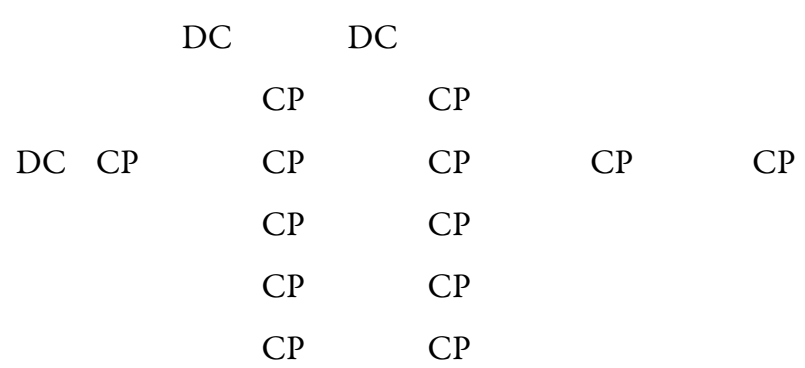

$\mathrm{CP}$

$\mathrm{CP}$

CP

CP

DC DC

$\mathrm{CP}$

$\mathrm{CP}$

$\mathrm{CP}$

CP

DC DC

$\begin{array}{lll} & \text { CP } & \text { CP } \\ & \text { DC CP } & \text { CP } \\ & & \text { CP }\end{array}$


Table 2 continued

\begin{tabular}{|c|c|c|c|c|c|c|c|c|c|c|c|c|}
\hline \multirow[t]{2}{*}{ Adverse reaction HLGT (bold) and LLT } & \multicolumn{2}{|l|}{ US } & \multicolumn{2}{|l|}{ UK } & \multicolumn{2}{|c|}{ IRE } & \multicolumn{2}{|c|}{ AUS } & \multicolumn{2}{|l|}{$\mathbf{N Z}$} & \multicolumn{2}{|c|}{ CAN } \\
\hline & $\mathrm{DL}$ & MG & $\mathrm{DL}$ & MG & $\overline{D L}$ & MG & DL & MG & DL & MG & DL & MG \\
\hline \multicolumn{13}{|l|}{ Peripheral neuropathies } \\
\hline Difficulties raising shoulders ${ }^{\mathrm{n}}$ & & & & $\mathrm{CP}$ & & $\mathrm{CP}$ & & & & & & \\
\hline Neuropathy & & & DC & $\mathrm{CP}$ & DC & $\mathrm{CP}$ & & & & & & \\
\hline \multicolumn{13}{|l|}{ Seizures (including subtypes) } \\
\hline Convulsions/seizures (fit) & & & & & & & & $\mathrm{CP}$ & & $\mathrm{CP}$ & & \\
\hline
\end{tabular}

AUS Australia, CAN Canada, $C P$ consult physician, $D C$ discontinue drug, $D L$ drug label (or national equivalent), $H L G T$ MedDRA high level group term, IRL Ireland, LLT MedDRA lowest level term, $M G$ medication guide (or national equivalent), NEC not elsewhere classified, $N Z$ New Zealand, UK United Kingdom, US United States

a LLT disturbances of smell and taste

b LLT sensorineural hearing loss

c LLT hyperacusis

d LLT masked facies

e LLT cervical dystonia

${ }^{\mathrm{f}}$ LLT burning sensation

g LLT dysarthria

h LLT balance difficulty

i LLT eye movement disorder

j LLT numbness in face

k LLT tongue movement disturbance

${ }^{1}$ LLT neurogenic pain

m LLT muscle weakness

${ }^{n}$ LLT neuralgic amyotrophy

DLs of the UK, IRL, AUS, and NZ each describe abnormal dreams occurring in $\geq 1 / 10$ of prophylactic users. Intriguingly, this analysis finds a recommendation to DC at the onset of reactions within the HLGT sleep disorders and disturbances only in the DLs and MGs of the UK and IRL. In contrast, the MG in CAN provides no recommendation for such reactions and notes only that certain of these usually "do not cause people to stop taking the medicine". In further contrast, the MGs of three other countries-the US, AUS, and NZ-explicitly recommend only $\mathrm{CP}$ for such reactions. Unusually, the NZ MG lists abnormal or strange dreams as serious side effects requiring immediate CP. However, in contradiction to the $\mathrm{DL}$, which lists abnormal dreams as very common, the MG states that such serious side effects are rare.

For those other adverse reactions among the remaining six HLGTs for which there is also partial international agreement in corresponding prescribing and patient safety guidance, the DLs of the UK, IRL, AUS, and NZ each list their incidence as unknown, or do not specifically list the adverse reactions. In contrast, for the adverse reactions vertigo and dizziness, which are listed among the remaining two HLGTs cranial nerve disorders, and neurological disorders NEC, respectively, there is corresponding prescribing and patient safety guidance only in the DL and MG of a single country (US). The DLs of the UK, IRL, AUS, and NZ each describe vertigo and dizziness as 
Table 3 Country-specific mefloquine patient and prescribing guidance, number recommending DC or CP for one or more adverse reactions, and number and countries with corresponding guidance for both, by adverse reaction HLGT

\begin{tabular}{|c|c|c|c|c|}
\hline \multirow[t]{2}{*}{ Adverse reaction HLGT } & \multirow{2}{*}{$\begin{array}{l}\text { MG } \\
n\end{array}$} & \multirow{2}{*}{$\begin{array}{l}\text { DL } \\
n\end{array}$} & \multicolumn{2}{|c|}{ Corresponding MG and DL guidance } \\
\hline & & & $n(\%)$ & Countries \\
\hline Anxiety disorders and symptoms & 6 & 6 & $6(100)$ & US, UK, IRL, AUS, NZ, CAN \\
\hline Changes in physical activity & 6 & 6 & $6(100)$ & US, UK, IRL, AUS, NZ, CAN \\
\hline Depressed mood disorders and disturbances & 6 & 6 & $6(100)$ & US, UK, IRL, AUS, NZ, CAN \\
\hline Deliria (including confusion) & 6 & 6 & $6(100)$ & US, UK, IRL, AUS, NZ, CAN \\
\hline Disturbances in thinking and perception & 5 & 3 & $3(50)$ & US, UK, IRL \\
\hline Personality disorders and disturbances in behavior & 5 & 3 & $3(50)$ & US, UK, IRL \\
\hline Suicidal and self-injurious behaviors NEC & 5 & 3 & $3(50)$ & US, UK, IRL \\
\hline Neuromuscular disorders & 5 & 2 & $2(33)$ & UK, IRL \\
\hline Schizophrenia and other psychotic disorders & 2 & 3 & $2(33)$ & UK, IRL \\
\hline Sleep disorders and disturbances & 5 & 2 & $2(33)$ & UK, IRL \\
\hline Peripheral neuropathies & 2 & 2 & $2(33)$ & UK, IRL \\
\hline Cranial nerve disorders (excluding neoplasms) & 5 & 1 & $1(17)$ & US \\
\hline Neurological disorders NEC & 5 & 1 & $1(17)$ & US \\
\hline Mood disorder and disturbances NEC & 5 & 0 & $0(0)$ & \\
\hline Headaches & 4 & 0 & $0(0)$ & \\
\hline Movement disorders (including parkinsonism) & 4 & 0 & $0(0)$ & \\
\hline Neurological disorders of the eye & 4 & 0 & $0(0)$ & \\
\hline Psychiatric and behavioral symptoms NEC & 3 & 0 & $0(0)$ & \\
\hline Cognitive and attention disorders and disturbances & 2 & 0 & $0(0)$ & \\
\hline Dementia and amnestic conditions & 2 & 0 & $0(0)$ & \\
\hline Psychiatric disorders NEC & 2 & 0 & $0(0)$ & \\
\hline Seizures (including subtypes) & 2 & 0 & $0(0)$ & \\
\hline
\end{tabular}

AUS Australia, $C A N$ Canada, $C P$ consult physician, $D C$ discontinue drug, $D L$ drug label (or national equivalent), $H L G T$ MedDRA $^{\circledR}$ high level group term, IRL Ireland, $M G$ medication guide (or national equivalent), $N E C$ not elsewhere classified, NZ New Zealand, UK United Kingdom, US United States

common, occurring in $\geq 1 / 100-1 / 10$ of prophylactic users.

\section{Relevance of Findings}

Demand for MQ has been negatively impacted due to increased awareness of the drug's potential to cause lasting central nervous system toxicity [1]—an outcome which, despite numerous proposed pathophysiological mechanisms [17-20], remains poorly understood. Amidst losses in market share to safer and better tolerated antimalarial drugs, the innovator, Roche, has elected to withdraw 
Lariam-branded MQ from a number of additional countries besides the US and CAN; to include IRL, Germany, and Denmark [21]. Although there are concerns the drug may soon be withdrawn from other countries for similar reasons [22], MQ is likely to remain available internationally in generic forms for some time. This analysis provides important insights that may be relevant during patient counseling and in the consideration of potential future improvements to MQ safety guidance.

For example, as MQ prophylaxis is commonly prescribed for travelers, who by definition may be far from the prescribing physician or healthcare provider at the time that adverse reactions occur, CP may not be immediately feasible, delaying any potential recommendation to DC in the case of a particular psychiatric or neurologic adverse reaction for which the MG recommends only CP. Similarly, even in the event that CP is immediately available-such as by telephone or email-lack of explicit recommendation in an MG for a patient to seek CP for a particular adverse reaction may also delay any potential physician direction to DC should the patient not recognize its significance. A review of narrative reports of MQ adverse reactions may aid national drug regulators in determining whether the failed recognition of the significance of a particular adverse reaction identified in this analysis, or delays in CP related to travel, may have contributed to more serious events. Such a review may also aid regulators in determining whether such more serious events may have been potentially preventable through the inclusion of more explicit recommendations to $\mathrm{CP}$ or DC the drug at the onset of particular prodromal reactions-such as sleep disorders and disturbances-for which there is already partial international agreement in prescribing and patient safety guidance.

Current national guidelines for the use of MQ in malaria prophylaxis among travelers may be informed by current safety guidance in the national DL and MG. Differences in prescribing and patient safety guidance between countries may inform differential national recommendations for use of $\mathrm{MQ}$, and contribute to disagreement in the setting of common international guidelines. This analysis may aid as a starting point for developing consensus for international guidelines on the use of MQ in spite of significant international disagreement in the current safety guidance.

Similarly, disagreement in international prescribing and patient safety guidance may contribute to apparently contradictory situations, where similar patients from similar countries, who experience identical neurologic or psychiatric adverse reactions from MQ while traveling, are directed to take discordant actions in response. For example, under current guidance in country-specific MGs, travelers from the UK and IRL are directed to DC MQ at the onset of abnormal dreams, while travelers from AUS and NZ are directed merely to CP and not to DC at their onset. In contrast, travelers from the US are provided with no specific advice for actions to be taken at the onset of this very common adverse reaction, while travelers from CAN are advised that bad dreams are "usually mild" and "do not cause people to stop taking the medicine". Such discordance, while presumably reflecting markedly differing national risk-benefit decision making, may contribute to confusion among travelers and to country-specific guidance not being followed. This analysis is expected to aid in clearly identifying those classes of neurologic and psychiatric adverse reactions for which 
there is disagreement in international recommendations, permitting physicians and healthcare providers to appropriately emphasize national guidance.

\section{Limitations}

This analysis has a number of limitations. Significantly, this analysis is limited to a review of prescribing and patient safety guidance from only six developed countries. These countries were chosen based on their principal use of English and a shared common cultural and linguistic heritage. The measures of international agreement in prescribing and patient safety guidance from this analysis can, therefore, not be generalized to other countries, whose prescribing and patient guidance were not specifically reviewed.

In addition, this analysis relies on a subjective interpretation of the language in the various DLs and MGs. Although reflecting consensus opinion among the study authors, based on the imprecise nature of this language, and the systematic but arbitrary rules employed in this analysis, there may be reasonable disagreement by others as to whether a DL or MG should be interpreted as recommending $\mathrm{DC}$ or $\mathrm{CP}$ at the onset of a particular adverse reaction. This limitation reflects the disagreement that may be expected between individual prescribers and patients in interpreting recommendations in the DL and MG.

In certain cases, this analysis may also have assigned certain adverse reactions to an HLGT distinct from what might have been seemingly implied by the patient or prescribing guidance. For example, based on the use of the MedDRA to categorize particular listed adverse reactions, this analysis assigned many neurologic LLTs that might be considered most consistent with peripheral neuropathy-including pain and numbness-to the HLGT neurological disorders
NEC. However, both the UK and the IRL DL state, "[m]efloquine should be discontinued in patients experiencing symptoms of neuropathy, including pain, burning, tingling, numbness, and/or weakness in order to prevent the development of an irreversible condition [emphasis added]". This limitation reflects the fact that idiomatic language commonly used by patients, physicians, and other healthcare providers may not necessarily adhere to the standard MedDRA vocabulary [23].

\section{CONCLUSIONS}

MQ prescribing guidance in a growing number of countries now recommends DC at the onset of any neuropsychiatric adverse reaction. This analysis has identified certain common and very common neuropsychiatric adverse reactions to $\mathrm{MQ}$ for which DC is explicitly recommended and for which there is complete or partial international agreement these reactions be specifically listed in both prescribing and patient guidance.

The results of this analysis suggest opportunities for physicians in these countries to improve patient counseling by specifically emphasizing the need to DC at the onset of these adverse reactions. The results of this analysis also suggest opportunities for international drug regulators to clarify language in future updates to remaining MQ DLs and MGs to better reflect national risk-benefit considerations for continued use of the drug.

\section{ACKNOWLEDGMENTS}

The authors thank Mr. Andrew Bryce and Mr. Christian Frankenfeld for their assistance in reviewing early drafts of this manuscript. No specific funds were allocated or received for this 
study. The article processing charges for this publication were funded by the authors. Dr. Nevin conceived of the study and drafted the final submitted manuscript. Ms. Byrd and Dr. Nevin analyzed and interpreted data, and critically reviewed and approved the final submitted manuscript. All named authors meet the International Committee of Medical Journal Editors (ICMJE) criteria for authorship for this manuscript, take responsibility for the integrity of the work as a whole, and have given final approval for the version to be published.

Disclosures. Remington L. Nevin has been retained as consultant and expert witness in legal cases involving claims of antimalarial drug toxicity. Aricia M. Byrd reports no conflicts of interest.

Compliance with Ethics Guidelines. This article is based on previously conducted studies and does not involve any new studies of human or animal subjects performed by any of the authors.

Open Access. This article is distributed under the terms of the Creative Commons Attribution-NonCommercial 4.0 International License (http://creativecommons.org/licenses/ by-nc/4.0/), which permits any noncommercial use, distribution, and reproduction in any medium, provided you give appropriate credit to the original author(s) and the source, provide a link to the Creative Commons license, and indicate if changes were made.

\section{REFERENCES}

1. Nevin RL. Idiosyncratic quinoline central nervous system toxicity: historical insights into the chronic neurological sequelae of mefloquine. Int J Parasitol Drugs Drug Resist. 2014;4:118-25.
2. Overbosch D, Schilthuis $H$, Bienzle $U$, et al. Atovaquone-proguanil versus mefloquine for malaria prophylaxis in nonimmune travelers: results from a randomized, double-blind study. Clin Infect Dis. 2001;33:1015-21.

3. Schlagenhauf $\mathrm{P}$, Tschopp A, Johnson $\mathrm{R}$, et al. Tolerability of malaria chemoprophylaxis in non-immune travellers to sub-Saharan Africa: multicentre, randomised, double blind, four arm study. BMJ. 2003;327:1078.

4. Stürchler D, Handschin J, Kaiser D, et al. Neuropsychiatric side effects of mefloquine. N Engl J Med. 1990;322:1752-3.

5. Nevin RL. Limbic encephalopathy and central vestibulopathy caused by mefloquine: a case report. Travel Med Infect Dis. 2012;10(3):144-51.

6. Ringqvist $\AA$, Bech P, Glenthøj B, Petersen E. Acute and long-term psychiatric side effects of mefloquine: a follow-up on Danish adverse event reports. Travel Med Infect Dis. 2015;13:80-8.

7. Teva. U.S. mefloquine product insert, June 2013.

8. F. Hoffman-La Roche. U.S. Lariam product information, November 1989.

9. F. Hoffman-La Roche. U.S. Lariam product information, July 2002.

10. U.S. Food and Drug Administration. FDA drug safety communication: FDA approves label changes for antimalarial drug mefloquine hydrochloride due to risk of serious psychiatric and nerve side effects, 29 July 2013. http://www. fda.gov/Drugs/DrugSafety/ucm362227.htm. Accessed 27 March 2016.

11. European Medicines Agency. Updated PRAC rapporteur assessment report on the signal of permanent neurologic (Vestibular) disorders with mefloquine. EMA/63963/2014, 31 January 2014.

12. U.S. Food and Drug Administration. Prescription drug product labeling; medication guide requirements-FDA. Final rule. Fed Regist. 1998;63:66378-400.

13. F. Hoffman-La Roche. U.S. Lariam dear health care professional letter, September 2003.

14. Brown EG, Wood L, Wood S. The medical dictionary for regulatory activities (MedDRA). Drug Saf. 1999;20(2):109-17.

15. U.S. Department of Health and Human Services. Docket No. FDA-2011-N-0411. Fed Regist. 2011;76:33310-4. 
16. Health Canada. Drugs and Health Products. Drug product database online query, 2015. http:// webprod5.hc-sc.gc.ca/dpd-bdpp/start-debuter.do. Accessed 27 March 2016.

17. Mawson A. Mefloquine use, psychosis, and violence: a retinoid toxicity hypothesis. Med Sci Monit. 2013;19:579-83.

18. Quinn JC. Complex membrane channel blockade: a unifying hypothesis for the prodromal and acute neuropsychiatric sequelae resulting from exposure to the antimalarial drug mefloquine. J Parasitol Res. $2015 ; 2015: 368064$.

19. Croft AM, Herxheimer A. Adverse effects of the antimalaria drug, mefloquine: due to primary liver damage with secondary thyroid involvement? BMC Public Health. 2002;2:6.
20. Nevin RL. Mefloquine neurotoxicity and gap junction blockade: critical insights in drug repositioning. Neurotoxicology. 2011;32:986-7.

21. Arznei-Telegramm. Quiet end to the anti-malarial drug mefloquine. Arznei-Telegramm. 2016;47:31-2.

22. Hawkes N. Falling demand for mefloquine threatens future of this important antimalarial, experts warn. BMJ. 2016;352:i1190.

23. Inácio $\mathrm{P}$, Airaksinen $\mathrm{M}$, Cavaco A. Language does not come "in boxes": assessing discrepancies between adverse drug reactions spontaneous reporting and MedDRA $^{\circledR}$ codes in European Portuguese. Res Social Adm Pharm. 2015;11:664-74. 